LBNL-45295

UCB-PTH-00/09

hep-ph/0003170

March 2000

\title{
The Higgs Mass and New Physics Scales in the Minimal Standard Model
}

\author{
Christopher Kolda and Hitoshi Murayama \\ Department of Physics \\ University of California \\ Berkeley, CA 94720, USA \\ and \\ Theory Group \\ Lawrence Berkeley National Laboratory \\ Berkeley, CA 94720, USA
}

\begin{abstract}
We study the theoretical correlation between the Higgs mass of the minimal standard model and the scale at which new physics is expected to occur. In addition to the classic constraints of unitarity, triviality and vacuum stability, we reexamine the constraints imposed by the precision electroweak data. We then pay particular attention to the constraint imposed by the absence of finetuning in the Higgs mass parameter (the Veltman condition). We find that the fine-tuning condition places a significant constraint on the new physics scale for the Higgs mass range $100 \mathrm{GeV} \lesssim m_{h} \lesssim 200 \mathrm{GeV}$ mostly unconstrained by the classic constraints.
\end{abstract}

${ }^{*}$ This work was supported in part by the Department of Energy under contract DE-AC0376SF00098 and in part by the National Science Foundation under grant PHY-95-14797. 


\section{Introduction}

The Standard Model of particle physics has passed every experimental challenge to date, the only important exception being neutrino oscillations. The gauge sector, namely the interactions of quarks and leptons with gauge bosons, has been verified at the permille level due mostly to the LEP and SLC experiments [1]. The Yukawa sector is being tested vigorously by exploiting flavor-changing and CP-violating phenomena. The last year has produced two new manifestations of $\mathrm{CP}$ violation [2, 3] a quarter century after the first evidence, and higher luminosity $B$-experiments will explore this sector even further. Yet the most mysterious and the least understood sector is the electroweak symmetry breaking sector. In the minimal standard model, our lack of understanding is parameterized by the mass of the Higgs boson, $m_{h}$.

There is actually another unknown parameter of the standard model. It is commonly assumed that the minimal standard model is merely a low-energy effective theory of some more fundamental theory which explains the origin of electroweak symmetry breaking. Such a theory may also explain the structure of fermion masses and mixings, the strange assignments of fermion gauge charges, and so forth. The energy scale, $\Lambda$, of such a (more) fundamental theory provides an ultraviolet cutoff for the standard model but is completely unknown at present.

In the absence of direct experimental information, theoretical considerations are our only guide to constraining the ranges of $m_{h}$ and $\Lambda$. An important example of such an aid is the electroweak precision analysis, where the precise measurements of masses, widths and asymmetries indirectly constrain the Higgs mass and new physics through loop diagrams. In this letter, we extend such analyses to determine the possible range of $m_{h}$ and $\Lambda$ based on various theoretical requirements. These include the traditional analyses of unitarity [4], triviality [5] and vacuum stability [6]. We will revisit the precision electroweak analysis of Hall and Kolda [7] using improved methodology and newer data. And finally, we will impose naturalness/fine-tuning constraints on the Higgs sector. We will find that naturalness may not be as constraining as is generally believed, but combined with the other requirements will provide a narrow region of interest in the search for new physics. This will be important in planning future high-energy experiments.

This type of discussion can be used in two different ways. If one picks a particular candidate for new physics with an associated energy scale $\Lambda$, a certain range of Higgs mass is preferred; thus we predict $m_{h}$ given a framework. On the other hand, if we find and measure the mass of the Higgs boson, this analysis provides an upper bound on the new physics scale $\Lambda$. Either way, the constraints on $\left(m_{h}, \Lambda\right)$ will be valuable.

The new physics at scale $\Lambda$ should protect the smallness of the electroweak scale $v=175 \mathrm{GeV}$ against the corrections due to physics at the Planck scale, $M_{P l}=$ $10^{19} \mathrm{GeV}$. The most often discussed candidate for such physics is probably supersymmetry (SUSY) (see, e.g., [8] for a review), but other possibilities (e.g., a composite Higgs sector [9, 10]) also exist. An alternative possibility is that the Planck-scale 
is actually much lower than apparent because of large extra dimensions 11]. Our analysis avoids specifying such new physics explicitly, and we try to remain as modelindependent as possible. This unfortunately introduces an uncertainty in the definition of the scale $\Lambda$. However for most choices of new physics, $\Lambda$ represents roughly the scale of some particle which has been integrated out to produce the standard model. This is of course the scale most relevant to direct searches at high energy colliders.

This letter is organized as follows. In the sections that follow, we explain the theoretical requirements on $m_{h}$ and $\Lambda$, starting with the well-known constraints coming from unitarity, triviality and vacuum stability. We then extend these to include the now-powerful arguments coming from the precision electroweak analysis. Finally we revisit an old idea in new clothing, namely the absence of fine-tuning and the so-called Veltman condition [12]. Finally we comment on the special case of supersymmetry briefly.

\section{The Usual Suspects}

Unitarity, triviality and vacuum stability are the classic triumvirate of Higgs mass constraints. Over the years, these three ideas have repeatedly guided our thinking about Higgs physics and scales of new physics, and are essential parts of any such discussion. But since they are so well-known we will only give a short discussion of them here.

\section{$2.1 \quad$ Unitarity}

Unitarity is simultaneously the strongest and weakest constraint when considering the Higgs mass and new physics [4]. It is the strongest in that it provides an upper bound on $m_{h}$ above which the standard Model is known to become non-perturbative. It is the weakest because it provides only binary information about that new physics: if $m_{h}$ exceeds its unitarity bound, then the new physics must appear almost immediately; if $m_{h}$ is less than its unitarity bound, then there is no need for new physics and there is nothing further to be said.

In this context, "unitarity" should more properly be called "tree-level unitarity" because it is the requirement that the tree-level contribution to the first partial wave in the expansion of various scattering amplitudes not exceed the unitarity bound. This breakdown of unitarity at the lowest order can either be cancelled by some new physics which plays the role of the Higgs, or by a cancellation of the tree-level against higher orders. The first implies new physics prima facie. The second implies a breakdown in perturbation theory at a nearby scale, which we will consider to be in itself "new physics" for the purposes of this article.

The calculation is simple and well-known (we follow the second paper in Ref. [4]), but helps to define our notation. Begin with the tree-level Higgs potential:

$$
V_{0}=\mu^{2}|\phi|^{2}+\lambda|\phi|^{4} \text {. }
$$


This potential satisfies the well-known minimization condition $|\phi|^{2}=-\mu^{2} / 2 \lambda$. Expanding $\phi$ about $v=175 \mathrm{GeV}$ in terms of the physical Higgs and Nambu-Goldstone bosons,

$$
\phi=\left(\begin{array}{c}
G^{+} \\
v+\frac{1}{\sqrt{2}}\left(h+i G^{0}\right)
\end{array}\right),
$$

yields $m_{h}^{2}=-2 \mu^{2}=4 \lambda v^{2}$ and all the Nambu-Goldstones are massless.

In scattering processes of gauge and Higgs bosons (at $\sqrt{s} \gg m_{W}$ ), the amplitudes can be decomposed into partial waves. Replacing the longitudinal components of the gauge bosons with Nambu-Goldstone bosons via the equivalence theorem [13], that decomposition takes the form:

$$
a_{J}(s)=\frac{1}{32 \pi} \int d(\cos \theta) P_{J}(\cos \theta) \mathcal{M}(s, \theta)
$$

for $a_{J}$ the $J$-th partial wave, $P_{J}$ the $J$-th Legendre polynomial and $\mathcal{M}$ the scattering matrix element. Recalling the scattering unitarity circle, one immediately notes that while $\left|\operatorname{Im} a_{J}\right| \leq 1$, the constraint on the real part is stronger:

$$
\left|\operatorname{Re} a_{J}\right| \leq \frac{1}{2}
$$

Now, in the absence of a physical Higgs boson, various Nambu-Goldstone scattering amplitudes grow with energy so that their tree-level amplitudes eventually exceed the unitarity bound. For example, the $J=0$ amplitude for $W_{L}^{+} W_{L}^{-} \rightarrow Z_{L} Z_{L}$ scattering is simply

$$
a_{J=0}(s)=\frac{s}{32 \pi v^{2}}-\frac{s}{32 \pi v^{2}} \frac{s}{s-m_{h}^{2}}+\mathcal{O}\left(\frac{m_{W}^{2}}{s}\right) \stackrel{s \gg m_{W}}{\longrightarrow} \frac{m_{h}^{2}}{32 \pi v^{2}}
$$

where the second term is due to Higgs exchange and cuts off the bad high energy behavior of the first term. The most divergent scattering amplitude is for the mixed $2 W_{L}^{+} W_{L}^{-}+Z_{L} Z_{L}(I=0)$ channel. Here $\llbracket$

$$
a_{J=0} \longrightarrow-\frac{5 m_{h}^{2}}{64 \pi v^{2}}
$$

which is already real. Bounding this by $\frac{1}{2}$ yields

$$
m_{h}<780 \mathrm{GeV} \text {. }
$$

Without a Higgs, the amplitude grows as $a_{J=0}=s / 32 \pi v^{2}$, so that the theory violates the unitarity bound at $\Lambda_{U}=1.2 \mathrm{TeV}$; that is, either new physics must appear or the theory will become strongly coupled at $\Lambda_{U}$. The bound on $\Lambda_{U}$ is slightly weaker than that on $m_{h}$ only because it is more general; the Higgs cancellation is the special case in which the new physics is the Higgs itself.

So, in summary, unitarity tells us that either $(i)$ the Higgs is lighter than about $780 \mathrm{GeV}$, or $(i i)$ new physics must appear below $\Lambda_{U}=1.2 \mathrm{TeV}$. 


\subsection{Triviality}

The second classic constraint, triviality [5], derives its name from the observation in $\varphi^{4}$-theory that the quartic self-coupling $\lambda$ monotically increases as a function of the momentum scale $Q$, becoming non-perturbative at the so-called Landau pole, $\Lambda_{T}$. Thus any construction of $\varphi^{4}$-theory which is to remain perturbative at all scales must have $\lambda=0$ identically, thus rendering the theory "trivial." A similar observation can be made for the $\lambda$-coupling in the Higgs sector of the standard model, with one important exception: the renormalization group flow of $\lambda$ receives contributions with both signs. Namely,

$$
16 \pi^{2} \frac{d \lambda}{d \log Q}=24 \lambda^{2}-\left(3 g^{\prime 2}+9 g^{2}-12 y_{t}^{2}\right) \lambda+\frac{3}{8} g^{\prime 4}+\frac{3}{4} g^{\prime 2} g^{2}+\frac{9}{8} g^{4}-6 y_{t}^{4}+\cdots
$$

where $y_{t}$ is the top quark Yukawa coupling and the ellipsis represents contributions from small Yukawas and higher loops. The first term on the right side leads to the inflation of $\lambda$ in the ultraviolet, while the last term dominates contributions which drives $\lambda$ to asymptotic freedom. Once again, given $m_{h}$, all terms in the renormalization group equation (RGE) are known and this equation can be solved simultaneously with the RGEs for $g, g^{\prime}$ and $y_{t}$. For large Higgs masses, the first term in Eq. (8) dominates so that

$$
\Lambda_{T} \simeq Q \exp \left[\frac{2 \pi^{2}}{3 \lambda(Q)}\right]
$$

for any $Q<\Lambda_{T}$. Larger Higgs masses obviously lead to Landau poles at lower scales. Thus for any value of $\Lambda_{T}$, there is a corresponding maximum value of $m_{h}$ for which the theory remains perturbative at all scales below the cutoff.

In Figure 1, we show the maximum value of $\Lambda_{T}$ consistent with a given $m_{h}$, calculated using the full coupled 2-loop RGEs (upper shaded region is excluded). But how robust is this bound? Once we allow new physics at a scale $\Lambda$ into the picture, it is clear that there may appear additional operators that can contribute to the physical Higgs mass without similarly contributing to its 4-point interaction. Such new physics could increase or decrease the triviality constraint on $m_{h}$, or even eliminate it altogether [14]. Furthermore, $\Lambda$ and $\Lambda_{T}$ need not coincide exactly.

These considerations lead to two conclusions: first, the triviality bound cannot be considered some sort of model-independent upper bound on $m_{h}$ - it is merely a bound above which new physics near the Landau pole is implied. Second, the scale of that new physics, while being near the Landau pole, could be a few times larger. This last lesson is one that will be true throughout most of these discussions: the exact scale at which some new particle is produced can differ from the scale $\Lambda_{T}$ implied by triviality, vacuum stability or precision electroweak physics by $\mathcal{O}(1)$ coefficients. The good news is that one generally expects the new particles to be lighter than $\Lambda_{T}$ if the new physics is perturbative (consider the example of the $W$ mass versus $1 / \sqrt{G_{F}}$ ). 


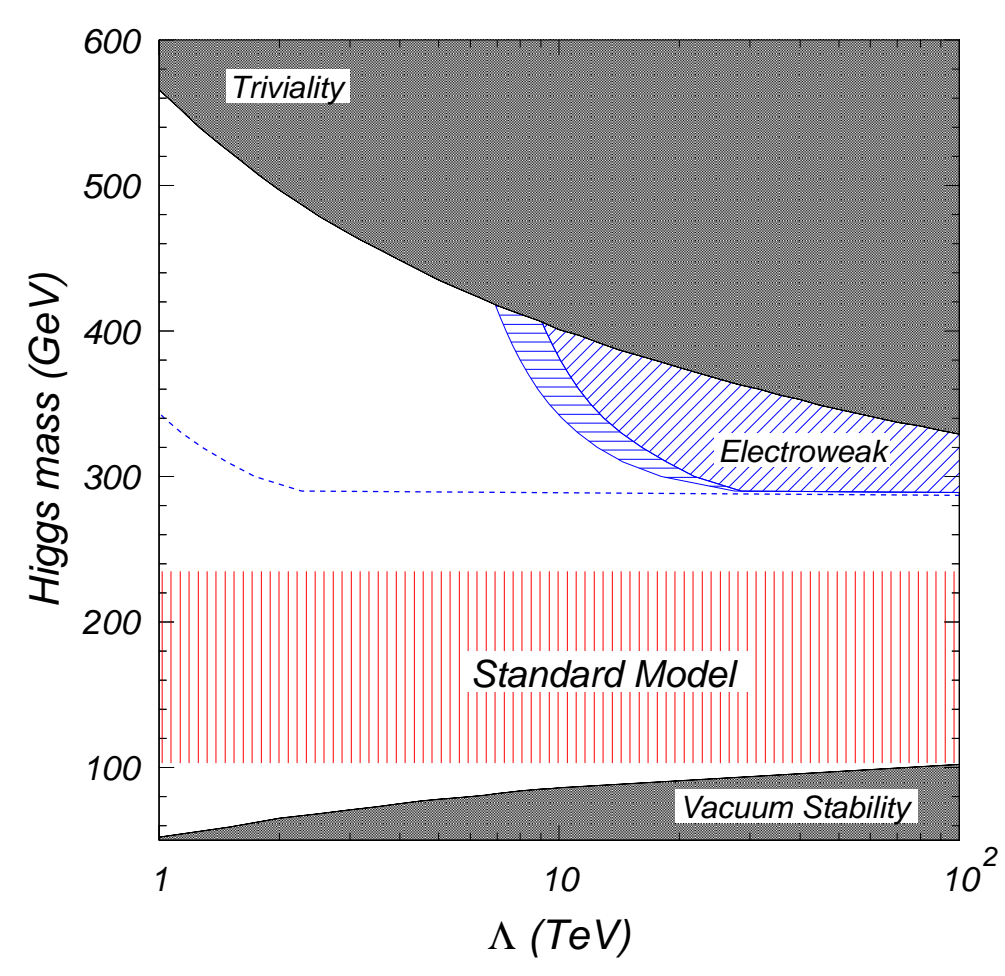

Figure 1: The classic constraints on the $m_{h}-\Lambda$ plane, including triviality (dark region at top) and vacuum stability (dark region at bottom). The hatched regions marked "Electroweak" and the region bounded by the dashed line are ruled out by precision electroweak analyses; see the text for details. The region marked "Standard Model" is allowed within the standard model, bounded by the LEP mass bound below and the $95 \%$ CL electroweak precision bound above.

\subsection{Vacuum Stability}

The third and final classic constraint, vacuum stability [6], can also be seen as coming (at a very simplified level) from the RGE for $\lambda$. Notice in Eq. (8) that if $m_{h}$ is very small then the top Yukawa contribution dominates, driving $\lambda$ negative in the ultraviolet. At the turn-over scale, $\Lambda_{V}$, the Higgs potential becomes unbounded from below and the Higgs vev falls off to infinity (or at least to $\phi \sim \Lambda_{V}$ ). Defining $\Lambda_{V}$ by $\lambda\left(\Lambda_{V}\right)=0$, and keeping only the top quark Yukawa:

$$
\Lambda_{V} \simeq Q \exp \left[\frac{8 \pi^{2} \lambda(Q)}{3 y_{t}^{4}(Q)}\right]
$$

for any $Q<\Lambda_{V}$.

A more complete and correct analysis requires the use of the renormalization group-improved effective potential. Rather than discussing the subtleties involved in such a calculation, we will simply quote the recent results of Casas et al. [15. Note 
that questions of tunnelling rates versus the lifetime of the universe have not been included in this result, but do not change the conclusions of the present paper at all.

One could also consider the effects coming from including higher dimensional operators in the potential [16] as we will do in the next section. These operators can have the effect of lifting the potential at large scales (e.g., $V \sim \phi^{6} / \Lambda^{2}$ ), or equivalently, shifting the value of $\lambda$ associated with a given Higgs mass. Whether the stability bound is raised or lowered depends sensitively on the sign of the new operators. To be conservative we would like to choose these signs to maximize the region allowed for the Higgs. But as we will see, the direct constraint coming from LEP makes this an unnecessary exercise.

In Figure 1, we show the maximum value of $\Lambda=\Lambda_{V}$ for a given value of $m_{h}$ (lower shaded region is excluded); alternatively, the plot can be interpreted as giving the minimum value of $m_{h}$ consistent with a given cutoff. Note that for the scales $\Lambda_{V}$ of interest to us here (those within a few orders of the weak scale), the current LEP bound of $m_{h}>102.6 \mathrm{GeV}(\sqrt{s}=196 \mathrm{GeV})$ [17] already supersedes the bounds coming from vacuum stability arguments. And thus there is also nothing to be gained by weakening the bound through the addition of higher dimensional operators.

\section{Precision Electroweak Constraints}

While triviality and vacuum stability only provide bounds on the Higgs mass as a function of the scale of new physics, a more direct constraint arises from precision electroweak measurements. A global fit of the data from the $Z$-pole experiments (ALEPH, DELPHI, L3, OPAL, SLD), from $\nu-N$ deep inelastic scattering experiments (NuTeV, CHARM, CCFR, CDHS), from low-energy $\nu$ - $e$ scattering experiments (CHARM II), from atomic parity violation experiments (on cesium and thallium), and from top quark production (CDF, D0) provides very strong constraints on the range of $m_{h}$ under the assumption that there is no physics other than that of the standard model near to the weak scale. The fits to $m_{h}$ are constrained primarily by the oblique corrections, and thus we can frame our discussion in terms of the usual $S$ and $T$ parameters [18]. (For an up-to-date discussion of the Higgs mass and the oblique parameters, see 19. We will set $U=0$ for this analysis since most types of new physics contribute little if anything to $U$.)

Assuming no physics beyond the standard model, one finds [19, 20]:

$$
m_{h}=\left(98_{-38}^{+57}\right) \mathrm{GeV}
$$

or a one-sided limit of $m_{h}<235 \mathrm{GeV}$ at $95 \% \mathrm{CL}$. What happens once new physics is included? Because the standard model already works so well, any deviations from it can be expressed using effective Lagrangian techniques, i.e., in terms of an expansion in inverse powers of the cutoff, $\Lambda$. All independent dimension- 6 operators which are consistent with the symmetries and particle content of the standard model have 
been catalogued [21] and their contributions to $S$ and $T$ have been studied [22]. Two operators can produce shifts in $S$ and $T$ so as to significantly alter the usual electroweak extraction of $m_{h}$ :

$$
\begin{gathered}
\mathcal{O}_{B W}=\frac{f_{B W}}{\Lambda^{2}} g g^{\prime}\left(\phi^{\dagger} \tau^{a} W_{\mu \nu}^{a} \phi\right) B^{\mu \nu} \longrightarrow \Delta S=-16 \pi \frac{v^{2}}{\Lambda^{2}} f_{B W} \\
\mathcal{O}_{\phi}=\frac{f_{\phi}}{\Lambda^{2}}\left(\phi^{\dagger} D_{\mu} \phi\right)\left(D^{\mu} \phi^{\dagger} \phi\right) \longrightarrow \Delta T=-\frac{1}{\alpha} \frac{v^{2}}{\Lambda^{2}} f_{\phi}
\end{gathered}
$$

where $\alpha$ is the fine-structure constant and $f_{B W}, f_{\phi}$ are unknown coefficients. Because the dependence of $S$ and $T$ on $m_{h}$ is primarily or exclusively logarithmic, small changes in their experimentally preferred values result in exponentially large shifts in $m_{h}$. Thus the operators $\mathcal{O}_{B W}$ and $\mathcal{O}_{\phi}$ can greatly affect the extraction of $m_{h}$ even for relatively large $\Lambda$ [7].

Of course, the correspondence between $m_{h}$ and $\Lambda$ is intimately tied to the values of $f_{B W}$ and $f_{\phi}$. These coefficients are in turn derived from the physics at $\Lambda$ which we do not know. Therefore we will consider three possible scenarios for each $\mathcal{O}_{i}$ $(i=\phi, B W)$ :

Loop-level: Physics at $\Lambda$ is perturbative and $\mathcal{O}_{i}$ is generated via loops. Therefore $\left|f_{i}\right| \leq \frac{1}{16 \pi^{2}}$

Tree-level: Physics at $\Lambda$ is perturbative but $\mathcal{O}_{\phi}$ is generated at tree-level: $\left|f_{\phi}\right| \leq 1$. $\mathcal{O}_{B W}$ however cannot be generated at tree-level [23], so $\left|f_{B W}\right| \leq \frac{1}{16 \pi^{2}}$.

Strong-coupling: Physics at $\Lambda$ is strongly coupled and all or some of the fields in $\mathcal{O}_{i}$ are composite. Naive dimensional analysis combined with custodial symmetry breaking tell us that $\left|f_{i}\right| \leq 1$.

These three cases were chosen because they represent the three most interesting limits of how new physics might affect $S$ and $T$ : the contributions to both are large $~ 1$, small $\sim \frac{1}{16 \pi^{2}}$, or the contributions to $T$ and much larger than those to $S$ (such as occurs in the simplest $Z-Z^{\prime}$ mixing models). The missing fourth case in which the contributions to $S$ dominate over those to $T$ occurs in only a few examples of which we know and usually (if not always) $S$ has a sign which is inconsistent with the data.

This last case requires some comment (see [24 for a more complete discussion). If the Higgs field itself is a composite, then naive dimensional analysis [25] tells us that $f_{B W} \sim 1$ while $f_{\phi} \sim 16 \pi^{2}$. On the other hand, those same arguments would lead one to conclude that the top quark Yukawa $y_{t} \sim 4 \pi$. To avoid this (and other disasters), strongly coupled theories near the weak scale should approximately conserve custodial isospin, $I$, since the top quark mass is a $\Delta I=\frac{1}{2}$ operator. Since experimentally $y_{t} \simeq 1$, the suppression associated with a $\Delta I=\frac{1}{2}$ insertion must be $\sim \frac{1}{4 \pi}$. The operator $\mathcal{O}_{\phi}$ also breaks custodial isospin, but by $\Delta I=1$. Creating it requires two $\Delta I=\frac{1}{2}$ insertions, so we conclude that it will naturally be suppressed by $\frac{1}{16 \pi^{2}}$ over its naive value. Thus $\left|f_{\phi}\right| \sim 1$. 
Arguments can be made for other choices of the coefficients, however it seems to us that these choices cover the region of interest. Of course, it is possible that different operators in the effective Lagrangian can arise with very different coefficients. In fact, we are counting on that since we are setting all operators other than $\mathcal{O}_{B W}$ and $\mathcal{O}_{\phi}$ to zero, including flavor-violating operators that would generally force the cutoff above 10 to $100 \mathrm{TeV}$. If we included these operators at the same level as the two we are considering, new physics would always be too weak to affect the electroweak analysis. In fact, whenever one concentrates on new physics at scales below 10 to $100 \mathrm{TeV}$, one must demand (implicitly or explicitly) that the new physics respects the flavor symmetries of the standard model; the physics we are considering falls into this class.

One could consider other operators which, while protecting the flavor structure of the standard model, alter other aspects of the electroweak analysis, such as the coupling of $Z$-bosons to electrons or quarks [26, 228]. Barbieri and Strumia [26] have considered a "statistical ensemble" of models in which several (flavor-conserving) operators were added to the standard model. They found that over most of the phase space, non-universal corrections to the standard model were so tightly constrained that the new contributions to $S$ and $T$ were suppressed. While this is certainly true, we are considering the more conservative questions of how large $m_{h}$ could be, and how large $\Lambda$ could be consistent with a given $m_{h}$. Adding more operators at the scale $\Lambda$ with fixed coefficients will, over the great majority of parameter space, only serve to rule out regions which we find to be allowed. It is possible that by carefully tuning the other operators, one could actually allow regions which we disallow, but this seems to us to be highly unlikely. Thus we feel that this analysis is as conservative as one could reasonably demand.

However, to be conservative, we must not impose correlations on the $f_{i}$ which may be unnatural. Ideally, one would like to set only an upper bound on each operator and allow them to vary independently within their allowed ranges so as to maximize $\Lambda$ for a given $m_{h}$. So for example, the strong-coupling case in which $\left|f_{i}\right| \leq 1$ really does mean that any $f_{i}$ in the range $-1 \leq f_{\phi} \leq 1$ and $-1 \leq f_{B W} \leq 1$ is allowed. One side-effect of this method is that there is no lower bound on $\Lambda$ as was found in [7]. However, it is easy to prove to oneself that for the purposes of maximizing the $m_{h}-\Lambda$ correlation, one is always forced to one of the corners of this rectangular parameter space where $f_{B W}= \pm 1$ and $f_{\phi}= \pm 1$ exactly. And because larger $m_{h}$ correspond to $S>0$ and $T<0$, we already know that we will be using the corner in which $f_{B W}=-f_{\phi}=+1$.

Analyses of this type have been completed by several groups [7, 26, 27]. The procedure we follow here is the same one that should be used once $m_{h}$ is measured. For each value of $m_{h}$ between 50 and $1000 \mathrm{GeV}$, the full range of electroweak data is fit to 2 unknown parameters, $S$ and T. A 95\% confidence region for $S$ and $T$ is defined to be the region in which $\chi^{2}-\chi_{\min }^{2}<5.99 . \Lambda$ is defined to be the maximum scale consistent with the values of $S$ and $T$ at any point inside this region, subject to the upper bounds placed on the $\left|f_{i}\right|$ by our choice of scenario (loop-level, tree-level, 
or strong-coupling). More specifically, for every point in the $(S, T)$ plane, there is a corresponding set of $(\Lambda(S), \Lambda(T))$ which are the maximum values of $\Lambda$ given the $f_{i}$ at their extremes derived from $S$ and $T$ respectively. Then for each point, we can define $\Lambda(S, T)=\min (\Lambda(S), \Lambda(T))$. The overall $\Lambda$ is then the maximum of the $\Lambda(S, T)$ within the $95 \%$ confidence region.

One side-effect of this procedure which appears to be unavoidable is that there exists a gap between the upper bound on $m_{h}$ in the standard model, and the lower bound on the region in which new physics can be excluded. Put another way, as $\Lambda \rightarrow \infty$, one would expect that all values of $m_{h}>235 \mathrm{GeV}$ would be excluded, since $235 \mathrm{GeV}$ represents the $95 \%$ bound on $m_{h}$ in the standard model. This is not the case for the following reason. In order to define regions which are allowed and disallowed, one must calculate $\chi_{\text {min }}^{2}$ within the assumed model and then allow $\chi^{2}$ to vary away from $\chi_{\min }^{2}$ by an amount consistent with the number of degrees of freedom in the model. The standard model analysis which generates a bound $m_{h}<235 \mathrm{GeV}$ produces a different $\chi_{\min }^{2}$ than the procedure which sets $m_{h}=235 \mathrm{GeV}$ and optimizes with respect to $S$ and T. Further, a 95\% upper bound on $m_{h}$ is defined by setting $\Delta \chi^{2}=2.71$, whereas the $95 \%$ region in the $(S, T)$ plane is defined using $\Delta \chi^{2}=5.99$. Thus one does not expect the two analysis to have the same limiting behaviors. Our conclusion is that, given current electroweak data, if the Higgs mass falls in the region $235 \mathrm{GeV} \leq m_{h} \leq 290 \mathrm{GeV}$, there will be clear evidence for the existence of new physics but no statistically significant statement can be made about what that new physics scale may be.

Having explained our procedure, let us present our results. They are summarized in Figure 1. The diagonally hatched area marked "Electroweak" represents the region ruled out for the "strong-coupling" class of models. The gap between the standard model bound and the new physics bound is clearly visible. For $\Lambda<10 \mathrm{TeV}$, the strongcoupling bound does no better than the triviality bound; however, once $\Lambda>10 \mathrm{TeV}$, the effects of the new operators become suppressed until finally at about $\Lambda \simeq 30 \mathrm{TeV}$, they are having no noticeable effect on the fit. The extension of this area which is horizontally hatched is the additional part of parameter space ruled out for the "tree-level" case.

Finally, the region above the dashed line is ruled out for the "loop-level" case. Here we notice that the new contributions to $S$ and $T$ are so small that they have no noticeable affect on the fits for $\Lambda \gtrsim 2.5 \mathrm{TeV}$, and even for $\Lambda=1 \mathrm{TeV}$ one can derive a bound $m_{h} \lesssim 340 \mathrm{GeV}$.

We can draw any number of conclusions from these limits. First, it is very hard to imagine that very weakly coupled physics $\left(f_{i} \sim \frac{1}{16 \pi^{2}}\right)$ is going to produce Higgs masses above the standard model limit. Thus any discovery of $m_{h} \gtrsim 290 \mathrm{GeV}$ is probably good evidence not only for new physics, but for physics with $\mathcal{O}(1)$ coefficients. Second, if $m_{h} \gtrsim 290 \mathrm{GeV}$, we can almost certainly conclude that there must be new physics at scales less than 10 to $30 \mathrm{TeV}$, depending on how heavy that Higgs is. For example, $m_{h} \gtrsim 400 \mathrm{GeV}$ requires new physics below $10 \mathrm{TeV}$. 


\section{Fine-Tuning}

Discussions of fine-tuning inevitably invite acrimony over definitions and degrees. There is a comfortable consensus that the mass parameter of the standard model Higgs sector is unstable to ultraviolet corrections and is thus fine-tuned. How to define that tuning is less precisely discussed, in part because one usually considers the cutoff to be far above the electroweak scale that precision in one's definitions seems hardly worthwhile. But we are considering physics very close to the weak scale, so we should take a moment to consider our definition.

Going back to the original Higgs potential and decomposition into physical and Nambu-Goldstone modes, notice that when we expand about some general vacuum with $\phi=v$ undetermined, the resulting masses are

$$
\begin{aligned}
m_{h}^{2} & =\mu^{2}+6 \lambda v^{2} \\
m_{\mathrm{NGB}}^{2} & =\mu^{2}+2 \lambda v^{2} .
\end{aligned}
$$

where $m_{G B}$ is the common mass of the Nambu-Goldstone bosons. At the minimum of the potential, $m_{h}^{2}=-2 \mu^{2}=4 \lambda v^{2}$ and all the Nambu-Goldstones are massless.

The issues we will be addressing concern the stability of the above minimum, and in particular the parameter $\mu^{2}$ to radiative corrections. And given that $m_{W}^{2}=g^{2} v^{2} / 2$, it is clear that the parameter $\mu^{2}$ is setting the electroweak scale, so we are really discussing the stability of the electroweak scale.

We study the radiative corrections by considering the one-loop contributions to the effective potential, which have the form:

$$
\begin{aligned}
V_{1}(\phi) & =\frac{1}{64 \pi^{2}} \int d^{4} k \mathrm{~S} \operatorname{Tr} \log \left(k^{2}+M^{2}(\phi)\right) \\
& =\frac{\Lambda^{2}}{32 \pi^{2}} \mathrm{~S} \operatorname{Tr} M^{2}(\phi)+\cdots
\end{aligned}
$$

where the supertrace is defined by $\mathrm{S} \operatorname{Tr}=\operatorname{Tr}(-1)^{F}$, and we are keeping only the quadratically divergent pieces. This new contribution can be absorbed into $V_{0}$ by shifting $\mu^{2}$ :

$$
\mu^{2} \longrightarrow \bar{\mu}^{2}=\mu^{2}+\frac{\Lambda^{2}}{32 \pi^{2} v^{2}} \mathrm{~S} \operatorname{Tr} M^{2}(\phi)=\mu^{2}+\frac{3 \Lambda^{2}}{32 \pi^{2} v^{2}}\left(2 m_{W}^{2}+m_{Z}^{2}+m_{h}^{2}-4 m_{t}^{2}\right) .
$$

(In these expressions and those that follow, we are only keeping the pieces of STr $M^{2}$ which are quadratic in $\phi$. . $^{2}$ This last expression is the source of the usual fine-tuning

${ }^{*}$ Naively, the supertrace is evaluated to be $6 m_{W}^{2}+3 m_{Z}^{2}+m_{h}^{2}-12 m_{t}^{2}$. However, there is one subtlety that must be recalled, namely that the trace is over the $\phi$-dependent masses, which for the scalar sector do not correspond to the physical masses. In particular,

$$
\sum_{\text {scalars }} M^{2}(\phi)=\left(\left.m_{h}^{2}\right|_{\phi=v}-\left.m_{h}^{2}\right|_{\phi=0}\right)+3\left(\left.m_{\mathrm{NGB}}^{2}\right|_{\phi=v}-\left.m_{\mathrm{NGB}}^{2}\right|_{\phi=0}\right)=\left.3 m_{h}^{2}\right|_{\phi=v}
$$

Thus Eq. (18) is reproduced. 
problem which arises within the SM; if $\Lambda \gg m_{W}$, then we must suppose that the tree-level $\mu^{2}$ and the loop contributions cancel to a very high precision in order for $\left|\bar{\mu}^{2}\right| \sim m_{W}^{2}$. There is one exception: Veltman famously noted [12 that there would be no (1-loop) fine-tuning problem if only $\mathrm{S} \operatorname{Tr} M^{2}$ turned out to be zero. We will refer to the statement that $\mathrm{S} \operatorname{Tr} M^{2}=0$ (and its higher order generalizations) as the "Veltman condition."

But while this condition STr $M^{2}=0$ is sufficient to cancel the 1-loop quadratically divergent contributions to $V$, it is not an all-orders result. In particular, the general form of $\bar{\mu}^{2}$ is given by [29]:

$$
\bar{\mu}^{2}=\mu^{2}+\Lambda^{2} \sum_{n=0}^{\infty} c_{n}\left(\lambda_{i}\right) \log ^{n}(\Lambda / Q)
$$

where $c_{0}=\left(32 \pi^{2}\right)^{-1} \mathrm{~S} \operatorname{Tr} M^{2} / v^{2}$ and the remaining $c_{n}$ can be calculated recursively via

$$
(n+1) c_{n+1}=\frac{d c_{n}}{d \log Q}=\beta_{i} \frac{\partial c_{n}}{\partial \lambda_{i}},
$$

from the requirement that the $\bar{\mu}^{2}$ should not depend on the renormalization scale $Q$. For example,

$$
\begin{aligned}
\left(16 \pi^{2}\right)^{2} c_{1}= & \lambda\left(144 \lambda-54 g^{2}-18 g^{\prime 2}+72 y_{t}^{2}\right)+y_{t}^{2}\left(27 g^{2}+17 g^{\prime 2}+96 g_{3}^{2}-90 y_{t}^{2}\right) \\
& -\frac{15}{2} g^{4}+\frac{25}{2} g^{\prime 4}+\frac{9}{2} g^{2} g^{\prime 2}
\end{aligned}
$$

Since each order in $n$ involves more factors of $\left(16 \pi^{2}\right)^{-1}$, the higher-loop contributions are decreasingly important.

The usual approach to the higher-order Veltman condition is to demand that each $c_{n}=0$ for all $n$ separately. In the infinite cutoff limit (or if one wants a cutoffindependent solution), this would be the correct procedure for solving the fine-tuning problem. And given that the various $c_{n}$ are independent, the set of conditions $c_{n}=0$ vastly overconstrains the inputs $\left(\lambda_{i}\right)$, so that no solution exists. Thus one is usually led to conclude [29] that the Veltman condition is ultimately not useful for solving the fine-tuning problem since it has no all-orders solution.

We would like to argue from a different limit. For $\Lambda$ not much larger than $m_{W}$, the stability of the weak scale does not require such a dramatic set of cancellations. This is easy to see. At 1-loop, stability of the weak scale is only threatened if $\Lambda \gtrsim 4 \pi v \simeq 2 \mathrm{TeV}$. Thus if new physics appears below about $2 \mathrm{TeV}$, it poses no threat to the weak scale even if the Veltman condition is not satisfied. (We will make this argument slightly more sophisticated shortly.) Now, were the 1-loop Veltman condition approximately satisfied (presumably by accident), then $\Lambda$ could be pushed up even higher. Two-loop quadratic divergences do not threaten the weak scale until $\Lambda^{2} \log \Lambda \gtrsim\left(16 \pi^{2}\right)^{2} v^{2}$; that is, $\Lambda \gtrsim 15 \mathrm{TeV}$. Thus if $m_{h}$ approximately satisfies the usual Veltman condition, one could push new physics off to scales $\sim 15 \mathrm{TeV}$ without tuning. 
This argument can be continued order by order. In particular, three-loop divergences are irrelevant until $\Lambda \gtrsim 50 \mathrm{TeV}$. Thus we could consider the possibility of scales up to $50 \mathrm{TeV}$ is the 2-loop Veltman condition were approximately satisfied. Since we are only interested in scales of this order, we will work to 2-loop order in the tuning condition.

This argument is slightly naive numerically, but it highlights the central truth that for cutoffs in the range that we might hope to probe any time in the foreseeable future: there is the possibility of suppressing tunings through approximate solutions to some finite-order version of the Veltman condition. For any such scale, the absence of large quadratic corrections is guaranteed simply by requiring

$$
\sum_{n=0}^{n_{\max }} c_{n}\left(\lambda_{i}\right) \log ^{n}\left(\Lambda / m_{h}\right)=0
$$

for some $n_{\max }$ chosen large enough so that the $\left(n_{\max }+1\right)$-order corrections are automatically small. For the purposes of this paper, we will set $n_{\max }=1$; that is, we will go 1-loop beyond the lowest order Veltman condition. From the arguments above, this is a perfectly acceptable procedure for studying cutoffs below roughly $50 \mathrm{TeV}$. Note that there is no guarantee that a solution to Eq. (22) even exists for large $n_{\max }$; however we will see that for $n_{\max }=1$ there is a solution.

Since this is such an interesting possibility, we will consider in more detail. Because all the parameters in the condition $\mathrm{S} \operatorname{Tr} M^{2}=0$ have been measured except for the Higgs mass, the Veltman condition predicts $m_{h}$. At LO,

$$
m_{h}=(317 \pm 11) \mathrm{GeV}
$$

where the uncertainty is dominated by the measurement of $m_{t}$ which we take to be $m_{t}=(174.3 \pm 5.1) \mathrm{GeV}$ [30] where the statistical and systematic errors are added in quadrature. At next-to-lowest order (NLO), the predicted Higgs mass decreases and becomes cutoff-dependent! This should not surprise us since the presence of the logarithms in Eq. (22) prevents any one value of $m_{h}$ from satisfying (22) at all scales. Another way to understand the $\Lambda$ dependence of the Veltman condition is the following: Since the renormalized parameter $\bar{\mu}^{2}$ in Eq. (19) does not depend on the renormalization scale $Q$, we can in particular take $Q=\Lambda$; then all logarithms vanish except for $n=0$. Therefore the Veltman condition is simply given by the lowest order expression $c_{0}\left(\lambda_{i}(\Lambda)\right)=0$. By rewriting the couplings $\lambda_{i}(\Lambda)$ in terms of the couplings which determine the Higgs mass $\lambda_{i}\left(m_{h}\right)$, we again find Eq. (22) and we understand easily why the Veltman condition depends on the cutoff $\Lambda$.

However, this "prediction" assumes exact cancellation of the quadratic divergences, which is not actually necessary if $\Lambda$ is close to the weak scale. This is another important difference that arises when one consider low cutoffs. Eq. (22) is more correctly written as an inequality:

$$
\sum_{n=0}^{n_{\max }} c_{n}\left(\lambda_{i}\right) \log ^{n}\left(\Lambda / m_{h}\right)<\frac{v^{2}}{\Lambda^{2}} .
$$


For $\Lambda=M_{P l}$, this implies a tuning of the Higgs mass (assuming any such value exists) to one part in $10^{16}$. Without a dynamical mechanism for generating such a well-tuned value, we have simply replaced the fine-tuning between bare and loop-generated scalar masses with an equally bad fine-tuning among the various couplings of the theory. Though we may have a prediction of $m_{h}$, the fine-tuning associated with that value is unacceptable. On the other hand, for low $\Lambda$, the tuning associated with any solution to Eq. (24) is much smaller; we will discuss this in more detail shortly.

In this paper we will be interested in deriving $\Lambda_{\max }$ as a function of $m_{h}$. Clearly, as $m_{h}$ approaches $317 \mathrm{GeV}$, the value of $\Lambda_{\max }$ will increase rapidly until higher order corrections cut it off; however we will find that $\Lambda_{\max }$ can still be well above the reach of current experiments for a wide range of $m_{h}$ centered around $317 \mathrm{GeV}$.

In order to continue, we must define more precisely what we mean by fine-tuning. Seemingly, the most natural definition would be the sensitivity of the weak scale to the cutoff: $\left|\delta m_{W}^{2}(\Lambda) / m_{W}^{2}\right|$, where $\delta m_{W}^{2}$ is the difference between the tree and loop values, with all other quantities held fixed. This is the measure of fine-tuning used in 31. Varying the weak scale as a function of $\Lambda$, we define

$$
\mathcal{F} \equiv\left|\frac{\delta m_{W}^{2}}{m_{W}^{2}}\right|=\left|\frac{\delta v^{2}}{v^{2}}\right|=\left|\frac{\delta \mu^{2}}{\mu^{2}}\right|=\left|\frac{\delta m_{h}^{2}}{m_{h}^{2}}\right|=\frac{2 \Lambda^{2}}{m_{h}^{2}}\left|\sum_{n} c_{n} \log ^{n}\left(\Lambda / m_{h}\right)\right| .
$$

For any particular value of $\mathcal{F}$, the weak scale is fine-tuned to one part in $\mathcal{F}$. In particular, $\mathcal{F} \leq 1$ represents the absence of tuning. Note that a light Higgs corresponds to small $\mu$ and thus greater fine-tuning as $\Lambda$ increases.

In Figure 2, we plot $\Lambda_{\max }$ as a function of $m_{h}$. The hatched regions represent various values of the tuning parameter: $\mathcal{F}>10$ (light hatching) and 100 (dark hatching), ignoring experimental uncertainties on $m_{t}$. Notice the light region extending to very large $\Lambda$. This is the line along which the Veltman condition is being approximately satisfied, and as expected, the value $m_{h}$ which satisfies the condition is scale-dependent.

We conclude three things from staring at Figure 2. First, if a light Higgs is discovered, then new physics is very near at hand. For example, for $m_{h}=130 \mathrm{GeV}$, finetuning of less than 1 part in 10 requires $\Lambda<2.3 \mathrm{TeV}$. The reason for this is simple: smaller $m_{h}$ means smaller $\mu$ which is all the harder to keep small. We notice secondly that for a generic heavy value of $m_{h}$, new physics must appear by about $3-5 \mathrm{TeV}$ in order to remain natural at the $10 \%$ level. Finally, for values of $m_{h}$ in the range of $200 \mathrm{GeV}$, the scale of new physics could turn out to be anomalously large, For example, $\Lambda$ can be as high as $10 \mathrm{TeV}$ if $m_{h}$ falls in the range $195 \mathrm{GeV}<m_{h}<215 \mathrm{GeV}$. We are not claiming that there exists a dynamical reason why $m_{h}$ would fall in this range, but if it did, our usual intuition about finetuning the electroweak scale would need modification.

\footnotetext{
${ }^{\dagger}$ We consider the square of the weak scale to be the fundamental quantity to be set by ultraviolet physics rather than the weak scale itself.
} 


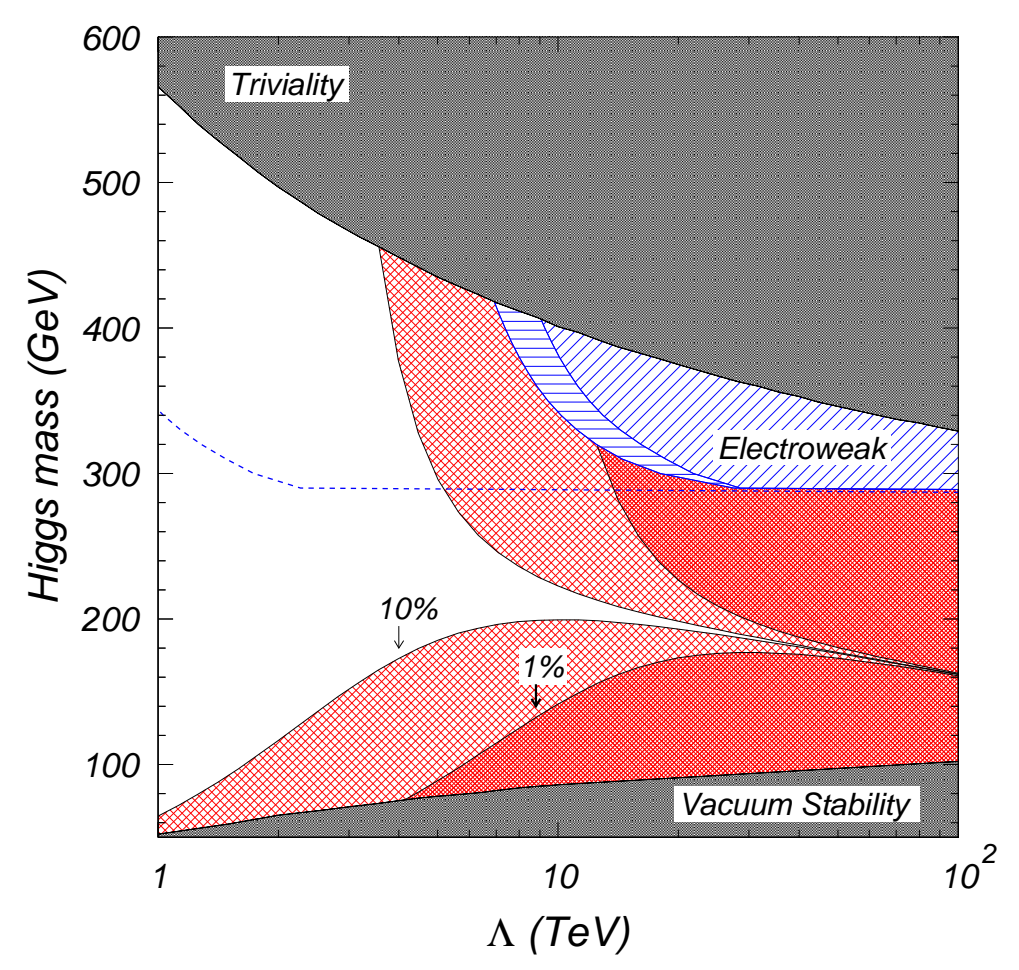

Figure 2: Plot in the $m_{h}-\Lambda$ plane showing the canonical constraints from Figure 11 as well as the tuning contours. The darkly hatched region marked "1\%" represents tunings of greater than 1 part in 100 ; the " $10 \%$ " region means greater than 1 part in 10 . The empty region is consistent with all constraints and has less than 1 part in 10 finetuning.

\section{The Supersymmetric Case}

After arguing that the scale of some general form of new physics could naturally sit at several $\mathrm{TeV}$, it is useful to consider a specific example, namely that of supersymmetry (SUSY). Usually when one considers SUSY models, one is led to conclude that finetunings of 1 part in 100 require the SUSY particle spectrum to lie below about $1 \mathrm{TeV}$ [31, 32]. Kane and King [33] have even argued for gluinos lighter than about $500 \mathrm{GeV}$ in some SUSY models in order to avoid tunings greater than 1 part in 10. Why is it, given the fact that SUSY is supposed to stabilize the weak scale by eliminating quadratic divergences, that the cutoff associated with SUSY so much smaller than those we have previously quoted?

The short answer is that the previous limits were for a general form of new physics; specific types may require much lower scales to avoid tunings. The long answer is that it is possible to arrange the parameters of SUSY so as to allow multi-TeV spectra without tunings, however in most models this behavior is not realized.

In SUSY models, one usually defines fine-tuning starting from the same point 
as above, namely the minimization of the Higgs potential. In SUSY, the treelevel minimization already depends explicitly on several masses in the problem. Specifically, one finds:

$$
\frac{1}{2} m_{Z}^{2}=\frac{m_{H_{1}}^{2}-m_{H_{2}}^{2} \tan ^{2} \beta}{\tan ^{2} \beta-1}-\mu^{2}
$$

where $H_{1}$ and $H_{2}$ are the two Higgs fields, $\tan \beta$ is the ratio of their vevs, and $m_{H_{1}}^{2}$, $m_{H_{2}}^{2}$ and $\mu$ are all mass parameters. From this minimization condition, there are clearly two ways to get equality: either all mass parameters on the righthand side are $\sim m_{W}^{2}$, or they are much larger but cancellations occur so that this particular function of their masses is $\sim m_{W}^{2}$. The first possibility is considered to be natural, representing no tuning. The second represents a tuning of roughly one part in $m_{Z}^{2} / \max \left(m_{H_{1,2}}^{2}, \mu^{2}\right)$. Since most models produce $m_{H_{1,2}}^{2} \sim \mu^{2} \sim M_{\mathrm{SUSY}}^{2}$, this translates into tunings of

$$
\mathcal{F} \simeq m_{Z}^{2} / M_{\mathrm{SUSY}}^{2}
$$

Note the absence of any loop suppression factors in this expression. It is this absence of additional $4 \pi$ suppressions that makes SUSY models seem more tuned at $1 \mathrm{TeV}$ than general models are at $5 \mathrm{TeV}$.

This comparison, however, is artificial. For the general models, we assume without argument that the tree-level mass term $\mu$ which sets the weak scale is naturally $\sim m_{W}$; we then only consider the effect of new physics through loops. In the usual SUSY case, we derive the weak scale from the SUSY scale already at tree-level, and so we are asking much more from our SUSY theory than in the more general case.

It is possible to make a more fair comparison. We could dictate that the mass parameters that enter the Higgs potential be weak-scale and then study their sensitivity to loop corrections coming from the remaining SUSY fields set at some higher mass scale $M_{\text {SUSY }}$. Because of the special properties of SUSY, the original quadratic divergences disappear, though they reappear in the guise of logarithmic divergences. As an example, the log-divergent 1-loop correction to $m_{H_{2}}^{2}$ is given by:

$$
\delta m_{H_{2}}^{2}=\frac{1}{16 \pi^{2}}\left(-6 g^{2} M_{2}^{2}-2 g^{\prime 2} M_{1}^{2}+6 y_{t}^{2}\left(m_{\tilde{t}_{L}}^{2}+m_{\tilde{t}_{R}}^{2}+m_{H_{2}}^{2}+A_{t}^{2}\right)\right) \log \Lambda
$$

where the contributions from gauginos, top squarks and $A$-terms are shown. Under most circumstances, this will be dominated by $m_{\tilde{t}_{L}} \simeq m_{\tilde{t}_{R}} \equiv M_{\text {SUSY }}$ so that

$$
\delta m_{H_{2}}^{2} \simeq \frac{3 y_{t}^{2}}{4 \pi^{2}} M_{\text {SUSY }}^{2} \log \Lambda .
$$

Except for the logarithm, this is exactly what we would expect for a more general theory. In SUSY models, the role of the cutoff $\Lambda$ is played by the scale at which SUSY is broken in the standard model sector. In the usual supergravity-type models, one takes $\Lambda \sim 10^{16-18} \mathrm{GeV}$, in which case the logarithm enhances the correction by about 
30. This 30 cancels the loop-suppression factor and we again conclude that SUSY must sit below about $1 \mathrm{TeV}$.

To those familiar with SUSY, nothing mysterious has happened here. We have simply restated the well-known fact that if we set the Higgs mass parameters to be much smaller than $M_{\text {SUSY }}$ at the cutoff, then the renormalization group flow down to the weak scale will tend to drag them back to $M_{\mathrm{SUSY}}$.

Several special cases have been discussed in the literature and we only mention them here. It is possible to arrange the spectrum at the cutoff scale so that the Higgs mass parameters are naturally driven to be small at the weak scale [34, but such a scenario requires tuning at the cutoff. We could alternatively choose to push a subset of the SUSY spectrum to the weak scale, keeping the rest heavy [35. Obviously, 1-loop naturalness is guaranteed if the top squarks and gauginos are $\sim m_{W}$. In such

a case 2-loop corrections [36] require that $M_{\mathrm{SUSY}} \lesssim 5-10 \mathrm{TeV}$ in line with our 1-loop naturalness bounds in the general model.

Finally, there is a limit in which SUSY models look exactly like the general case, but two requirements must be met: first, the cutoff itself must be approximately $M_{\text {SUSY }}$ in order to kill the logarithm; second, the underlying physics which sets the Higgs mass parameters must set them to be $\sim m_{W} \ll M_{\text {SUSY }}$ at lowest order. The first requirement is met, for example, in models with gauge-mediated SUSY-breaking, but we do not know how to simultaneously meet both. If both of these are met, loop corrections can be small and the cutoff (and SUSY with it) can be safely moved into the multi-TeV regime.

\section{Conclusions}

In this letter, we studied the theoretical constraints on the Higgs mass and new physics scales in the minimal standard model. In addition to the constraints coming from unitarity, triviality, vacuum stability and the electroweak precision analysis, we discussed the constraint from the absence of fine-tuning in the Higgs mass parameter (the Veltman condition). As is well-known, the classic constraints allow a very high scale of new physics for a Higgs in the mass range $100 \mathrm{GeV} \lesssim m_{h} \lesssim 200 \mathrm{GeV}$. However, the fine-tuning condition places a significant constraint on the new physics scale for this Higgs mass range. For most Higss mass values, we need new physics below 4 to $7 \mathrm{TeV}$ (15 to $20 \mathrm{TeV}$ ) if we tolerate $10 \%$ (1\%) fine-tuning. On the other hand, there is a narrow throat around $m_{h} \sim 200 \mathrm{GeV}$ which could allow much higher new physics scale. We noted, however, that a known specific example of new physics which avoids fine-tuning naturally (supersymmetry) is constrained even more tightly. Even though one cannot draw a strict numerical limit from these observations, the results presented here shed new light on the scale of physics beyond the minimal standard model. 


\section{Acknowledgements}

We would like to thank M. Chanowitz and L. Hall for many interesting discussions of the ideas presented here. This work was supported in part by the Department of Energy under contract DE-AC03-76SF00098 and in part by the National Science Foundation under grant PHY-95-14797.

\section{References}

[1] LEP Electroweak Working Group, http://www.cern.ch/LEPEWWG/

[2] T. Affolder et al. [CDF Collaboration], Phys. Rev. D61, 072005 (2000).

[3] A. Alavi-Harati et al. [KTeV Collaboration], Phys. Rev. Lett. 83, 22 (1999);

V. Fanti et al. [NA48 Collaboration], Phys. Lett. B465, 335 (1999).

[4] B. Lee, C. Quigg and H. Thacker, Phys. Rev. D16, 1519 (1977);

W. Marciano, G. Valencia and S. Willenbrock, Phys. Rev. D40, 1725 (1989).

[5] N. Cabibbo, L. Maiani, G. Parisi and R. Petronzio, Nucl. Phys. B158, 295 (1979);

M. Lindner, Z. Phys. C31, 295 (1986).

[6] For reviews, see M. Sher, Phys. Rept. 179, 273 (1989);

M. Quiros, hep-ph/9703412, in "Perspectives on Higgs Physics II," G. Kane, ed., World Scientific, Singapore (1998).

[7] L. Hall and C. Kolda, Phys. Lett. B459, 213 (1999).

[8] H. Murayama, lectures at the ITCP Summer School, hep-ph/0002232.

[9] L. Susskind, Phys. Rev. D20, 2619 (1979);

E. Farhi and L. Susskind, Phys. Rept. 74, 277 (1981).

[10] D. Kaplan, H. Georgi and S. Dimopoulos, Phys. Lett. B136, 187 (1984).

[11] N. Arkani-Hamed, S. Dimopoulos and G. Dvali, Phys. Lett. B429, 263 (1998).

[12] M. Veltman, Acta Phys. Polon. B12, 437 (1981).

[13] The first paper in 《4;

M. Chanowitz and M. K. Gaillard, Nucl. Phys. B261, 379 (1985).

[14] M. Chanowitz, private communication.

For a similar discussion on the vacuum stability constraint, see A. Datta and X. Zhang, Phys. Rev. D61, 074033 (2000). 
[15] J. Casas, J. Espinosa and M. Quiros, Phys. Lett. B382, 374 (1996).

[16] A. Datta, B. Young and X. Zhang, Phys. Lett. B385, 225 (1996).

[17] P. McNamara (for the LEP Higgs Working Group), talk given at the LEPC meeting, CERN, Sept. 7, 1999. See http://www.cern.ch/LEPHIGGS/talks/.

[18] M. Peskin and T. Takeuchi, Phys. Rev. Lett. 65, 964 (1990) and Phys. Rev. D46, 381 (1992);

G. Altarelli and R. Barbieri, Phys. Lett. B253, 161 (1991).

[19] J. Erler and P. Langacker, "Electroweak Model and Constraints on New Physics: in Review of Particle Physics," Eur. Phys. J. C3, 90 (1998);

K. Hagiwara, Ann. Rev. Nucl. Part. Sci. 48, 463 (1998).

[20] All electroweak fits were performed with the GAPP routines of J. Erler:

J. Erler and P. Langacker, "Status of the standard model," hep-ph/9809352.

See also http://wWw.physics.upenn.edu/ erler/electroweak/GAPP.html.

[21] W. Buchmüller and D. Wyler, Nucl. Phys. B268, 621 (1986).

[22] M. Golden and L. Randall, Nucl. Phys. B361, 3 (1991);

B. Holdom and J. Terning, Phys. Lett. B247, 88 (1990);

B. Holdom, Phys. Lett. B259, 329 (1991).

[23] C. Arzt, M. Einhorn and J. Wudka, Nucl. Phys. B433, 41 (1995).

[24] R. S. Chivukula and E. Simmons, Phys. Lett. B388, 788 (1996).

[25] H. Georgi, Phys. Lett. B298, 187 (1993).

[26] R. Barbieri and A. Strumia, Phys. Lett. B462, 144 (1999).

[27] R. S. Chivukula and N. Evans, Phys. Lett. B464, 244 (1999);

J. Bagger, A. Falk and M. Swartz, Phys. Rev. Lett. 84, 1385 (2000);

R. S. Chivukula, N. Evans and C. Hoelbling, hep-ph/0002022.

[28] T. Rizzo and J. Wells, Phys. Rev. D61, 016007 (2000);

A. Strumia, Phys. Lett. B466, 107 (1999).

[29] M. Einhorn and D. R. T. Jones, Phys. Rev. D46, 5206 (1992).

[30] M. Lancaster, talk presented at the XIX International Symposium on Lepton and Photon Interactions at High Energies, Stanford University, August 9-14, 1999, hep-ex/9912031.

[31] R. Barbieri and G. Giudice, Nucl. Phys. B306, 63 (1988). 
[32] G. Kane, C. Kolda, L. Roszkowski and J. Wells, Phys. Rev. D49, 6173 (1994).

[33] G. Kane and S. King, Phys. Lett. B451, 113 (1999).

[34] J. Feng, C. Kolda and N. Polonsky, Nucl. Phys. B546, 3 (1999);

J. Bagger, J. Feng and N. Polonsky, Nucl. Phys. B563, 3 (1999).

[35] A. Cohen, D. Kaplan, and A. Nelson, Phys. Lett. B388, 588 (1996).

[36] N. Arkani-Hamed and H. Murayama, Phys. Rev. D56, 6733 (1997). 